\title{
LA INVERSIÓN EN LA CAPACIDAD DEL CAPITAL HUMANO Y LA METODOLOGÍA DE LA GESTIÓN POR COMPETENCIAS
}

\author{
CAPACITY OF INVESTMENT IN HUMAN CAPITAL AND METHODOLOGY \\ FOR MANAGEMENT SKILLS \\ Guadalupe Esperanza Alvarado Daga* \\ Maestrista de la Unidad de Posgrado - Facultad de Ciencias Administrativas
}

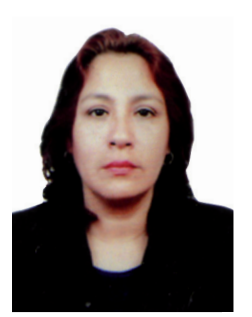

\author{
Universidad Nacional Mayor de San Marcos-UNMSM / Lima-Perú
}

[Recepción: Julio de 2013/ Conformidad: Agosto 2013]

\section{RESUMEN}

En un mundo globalizado, la empresa moderna necesita ir rumbo a la excelencia, lo cual implica que, debe estar preparada para competir con calidad con otras empresas del mismo sector industrial. Es importante, considerar al capital humano, como una posesión valiosa en la obtención de dicho propósito; para ello hay que dotarlo de las competencias adecuadas que, se adquieren a través de la capacitación por competencias considerada como una inversión continua. El día a día de una empresa depende de ello, por la influencia del desempeño de las actividades en los procesos que realiza este talento humano. El presente trabajo es una justificación y un aporte para la gestión del talento humano.

\section{Palabras Clave:}

Balance Scorecard, calidad, talento humano, capacitación por competencias.

\begin{abstract}
In a globalized world, the modern enterprise needs to go towards excellence, which means that you should be prepared to compete in quality with other companies in the same industry. It is important to consider human capital as a valuable asset in achieving this aim, for this we must equip them with the appropriate skills that are acquired through training competency considered as a continuous investment. The daily life of a company depends on it, by the influence of the performance of the activities in the processes that this human talent. The present work is a justification and a contribution to the management of human talent.
\end{abstract}

\section{Keywords:}

Balance Scorecard, quality, human talent, skills-based training.

* Ingeniera Química. Colegio de Ingenieros del Perú - Capítulo de Ingenieros Químicos. E-mail: lupe77ingquim@hotmail.com 


\section{INTRODUCCIÓN}

Este estudio tiene como propósito, proporcionar información sobre la aplicación de Balance Scorecard en la empresa peruana, así como establecer que la capacitación de las personas es una inversión y como tal, debe ser canalizada adecuadamente a través de una metodología de expertos. En este caso, según Martha Alles, permite seleccionar el personal adecuado para la capacitación por competencias; evaluando lo intangible, logrando la selección de las familias estratégicas para ser capacitadas, mejorando los procesos de la empresa y permitiendo llevar a cabo la alineación del Balanced Scorecard, resultando como consecuencia, la recuperación de la inversión, así como, la rentabilidad generada por dicha inversión. La limitación del presente estudio radica en el acceso a la información para atender una mayor cantidad de empresas y por ello se tuvo que recurrir a los casos comprobados y verificados que participaron en el Premio Nacional a la Gestión de la Calidad de los años 2012 y 2010.

\section{LA GESTIÓN POR COMPETENCIAS DEL TALENTO HUMANO}

En la gestión por competencias del talento humano se encuentra la Capacitación por Competencias como componente novedoso. En el Perú su aplicación ha dado buenos resultados en empresas de servicios, de producción y del sector público, aplicándolo en los aspectos financieros y por resultados como estrategia para incrementar la cuota de mercado, reducir los costos y asegurar la calidad del producto o servicio, contando siempre con la participación importante del equipo de trabajo que conforma la organización, es decir, las "personas".

La capacitación de las personas se realiza por diversos motivos como son: el de aumentar el mercado, mejorar la rentabilidad, disminuir las quejas de los clientes, minimizar fallas en el producto, etc., lo que permitirá a las empresas tomar esta decisión, presentándose estas interrogantes ¿A quiénes se deberá capacitar? ¿Será conveniente gastar en la capacitación del personal? ¿Cómo saber a quienes se debe capacitar? ¿Cómo proyectarnos para que la capacitación del personal sea una inversión y no un gasto? Según la metodología de Kaplan y Norton (2005) "la gestión empresarial ha dado resultados óptimos aplicando el Balance Scorecard o Cuadro de Mando Integral que plantea elaborar un mapa estratégico donde se describe la lógica de la estrategia de la empresa, mostrando claramente los objetivos de los procesos internos básicos que crean valor y los activos intangibles necesarios para respaldarlos. Los principios básicos de los mapas estratégicos son la perspectiva financiera (creación de valor para los accionistas), la perspectiva del cliente (proposición de valor diferenciada para el cliente), la perspectiva interna (procesos) y la perspectiva de aprendizaje y crecimiento (Talento Humano).

A través de la aplicación de la metodología sobre mapas estratégicos y Cuadro de Mando Integral, se establecieron las estrategias como relaciones causa - efecto, planteadas mediante cuatro perspectivas a saber: perspectiva financiera (creación de valor para los accionistas); perspectiva del cliente (proposición de valor diferenciada para el cliente), perspectiva interna (procesos) y perspectiva de aprendizaje y crecimiento.

Por otro lado, la alineación estratégica determinó el valor de los activos intangibles. La última perspectiva, describe los activos intangibles de la empresa y la función que tuvo en la estrategia. Se clasificaron en: a) Capital humano (habilidades, talentos y conocimientos de sus empleados), b) Capital de información (bases de datos, sistemas de información, redes e infraestructura tecnológica) y c) Capital organizativo (cultura, liderazgo, coordinación de los empleados, trabajo en equipo y gestión del conocimiento).

Esta metodología permitió mejorar la eficiencia de sus procesos, aumentar su productividad mediante la eliminación de gastos innecesarios, descubrir los cuellos de botella de los procesos, minimizar los tiempos de realización de sus actividades consiguiendo la satisfacción del cliente".

Pero si esta metodología orientó en cuanto a lo que se requiere para obtener una rentabilidad deseada, el alineamiento de aprendizaje y desarrollo direccionan a que es la apacitación por competencias

82/ QVIPURAMAYOC | Vol. 21(40) 2013 
la que se necesita para conseguirla. Una de las metodologías para evaluar las Competencias del Talento Humano enunciada por Martha Alles (2010) ha sido la más adecuada, a través de su obra "La Trilogía" ha proporcionado la experiencia para la aplicación de su metodología, realizada a empresas de todos los países hispanoparlantes, dando una visión regional relevante y el contacto profesional con empresas que plantean sus estrategias a mediano y largo plazo, resultando una perspectiva actualizada sobre el énfasis en la selección de las competencias más utilizadas. Esta obra está estructurada en la base de una explicación conceptual de las buenas prácticas y para su aplicación efectiva propuso 60 competencias más utilizadas detallando las descripciones, sus definiciones y apertura en niveles, agrupadas en cardinales: específicas gerenciales y específicas por área, proporcionando además, 1500 comportamientos relacionados con las competencias, complementando con un diccionario de preguntas para evaluar las competencias más utilizadas en el siglo XXI".

\section{RECONOCIMIENTO DE LA GESTION DE CALIDAD TENIENDO COMO BASE LA CA- PACITACIÓN POR COMPETENCIAS EN EL PERÚ}

En el Perú, la gestión de la calidad es reconocida a través del otorgamiento del Premio Nacional a la Calidad (CDI 2013), a las empresas que han demostrado ser muy eficientes, donde se incluye la Gestión del Talento Humano teniendo como base la capacitación continua del capital humano.

Este instrumento ha sido diseñado para orientar a las organizaciones en sus procesos de mejora continua, contribuyen de esta manera a incrementar sus condiciones de competitividad, el mismo que está respaldado por un Modelo de Excelencia (CDI 2013), en la gestión que corresponda a las buenas prácticas, a la cual se someten las organizaciones participantes. Este premio es otorgado por el Comité de Calidad a las organizaciones destacadas por sus logros al implementar el modelo el cual ha sido diseñado para llevar a cabo procesos de autoevaluación, postular al premio y retroalimentar a los postulantes, además de cumplir tres roles:
- Ayudar a mejorar las prácticas de desempeño organizacional, capacidad y resultados.

- Facilitar la comunicación y compartir información de las mejores prácticas entre las organizaciones.

- Servir como herramienta de trabajo para entender y manejar el desempeño y para guiar la planificación organizacional y las oportunidades de aprendizaje.

El Modelo (CDI 2013) está construido en base a la interrelación de los siguientes valores centrales y conceptos:

- Liderazgo visionario

- Excelencia orientada al cliente

- Aprendizaje personal y organizacional

- Valoración del personal y de los socios

- Agilidad y flexibilidad

- Orientación hacia el futuro

- Gestión de la innovación

- Gestión basada en hechos

- Responsabilidad social

- Orientación a resultados y creación de valor

- Perspectiva de sistema

Liderazgo visionario.- Consta del establecimiento por la Alta Dirección de la visión y la misión, valores de la organización, despliegue de la misión, visión y valores corporativos a través de su sistema de liderazgo a todos los trabajadores, proveedores y socios clave, compromiso con los valores de la organización.

Excelencia orientada al cliente.- Consiste en que la organización identifica e innova la oferta de productos para satisfacer los requisitos y pueda exceder las expectativas de sus grupos de clientes y segmentos de mercado (identificados en el Perfil Organizacional), identificación e innovación las ofertas de productos para captar nuevos clientes y proporcionar oportunidades para la expansión de las relaciones con los clientes.

Aprendizaje personal y organizacional.- El aprendizaje organizacional incluye tanto mejoras continuas de enfoques existentes como cambios o innovaciones significativas, dirigiéndose a nuevas metas y enfoques. 
Valoración del personal y de los socios.- Valorar a la gente significa comprometerse con su satisfacción, desarrollo y bienestar. Esto involucra prácticas de trabajo más flexible y de alto desempeño adecuadas a lugares de trabajo y necesidades familiares.

Las organizaciones necesitan construir relaciones internas y externas para facilitar el logro de las metas. Las relaciones internas pueden incluir cooperación en el trabajo y las relaciones externas pueden incluir clientes, proveedores y organizaciones de educación o de la comunidad.

Agilidad y flexibilidad.- El éxito en un ambiente siempre cambiante y competitivo a nivel global, demanda agilidad (capacidad para el cambio rápido) y flexibilidad. Un factor de éxito importante para afrontar retos competitivos es el tiempo de diseño o de ciclo de innovación.

Orientación hacia el futuro.- Asegurar la sostenibilidad de una organización requiere entender los factores que afectan a la organización y al mercado en el corto y largo plazo.

Gestión de la innovación.- La innovación debe dirigir a la organización a nuevas dimensiones de desempeño y no es sólo responsabilidad de departamentos de investigación y desarrollo; es importante para todos los aspectos de las operaciones de la organización y todos los sistemas y procesos de trabajo.

Gestión basada en hechos.- Las organizaciones dependen de la medición y el análisis del desempeño. La medición del desempeño debe incluir el desempeño del cliente, producto, servicio y proceso; comparaciones de desempeño operacional, de mercado y competitivo; desempeño del proveedor, del personal, del socio, de costos y financiero; así como resultados de gobierno y de cumplimiento.

Responsabilidad social.- Los líderes de una organización deben enfatizar las responsabilidades hacia el público, el comportamiento ético y la necesidad de considerar el beneficio y bienestar social.

Orientación a resultados y creación de valor.Los resultados deben ser usados para crear y balan- cear valor para las partes interesadas clave: clientes, personal, accionistas, proveedores, socios, el público y la comunidad.

Perspectiva de sistema.- Una perspectiva de sistema incluye el enfoque de la alta dirección en direcciones estratégicas y en los clientes. Significa que la alta dirección monitorea, responde y gestiona el desempeño basándose en los resultados. Una perspectiva de sistema también incluye utilizar las medidas, indicadores, competencias esenciales y conocimientos organizacionales para construir las estrategias clave.

\section{EMPRESAS GANADORAS DE LOS CUATRO ÚLTIMOS AÑOS SEGÚN CDI - 2013}

\section{Año 2012. Premio Nacional a la Calidad del Perú y Medalla líder en Calidad Ca- TEGoría Oro}

\section{Categoría Producción:}

AUSTRAL GROUP S.A.A.

Informe de Postulación (2012), demuestra que utiliza un Modelo de Capacitación por Competencias "Siendo una empresa pesquera líder mundial, con más de 25 años de experiencia realiza actividades en los países de Perú, Noruega, Escocia y Chile, promueve el desarrollo del Personal y de los Líderes con un Plan de Capacitación acorde a sus necesidades analizando sus brechas con información dada por el trabajador con el perfil que exige el puesto de trabajo. Estos resultados fueron revisados a nivel de jefaturas y gerencias. La información estadística de la capacitación por temas de interés para la empresa que recibieron los trabajadores desde el 2010 - 2012.

Es el caso de los tripulantes de las embarcaciones que recibieron cursos relacionados con la interiorización de los valores de la empresa, competencias organizacionales, liderazgo, conocimientos sobre procesos de la empresa, administración y organización. Evaluando los cursos de capacitación recibido después por los participantes a través de las encuestas así como posterior seguimiento de lo aprendido. 
Gráfico $\mathrm{N}^{\circ}$ 1. Total de Capacitaciones por Temas

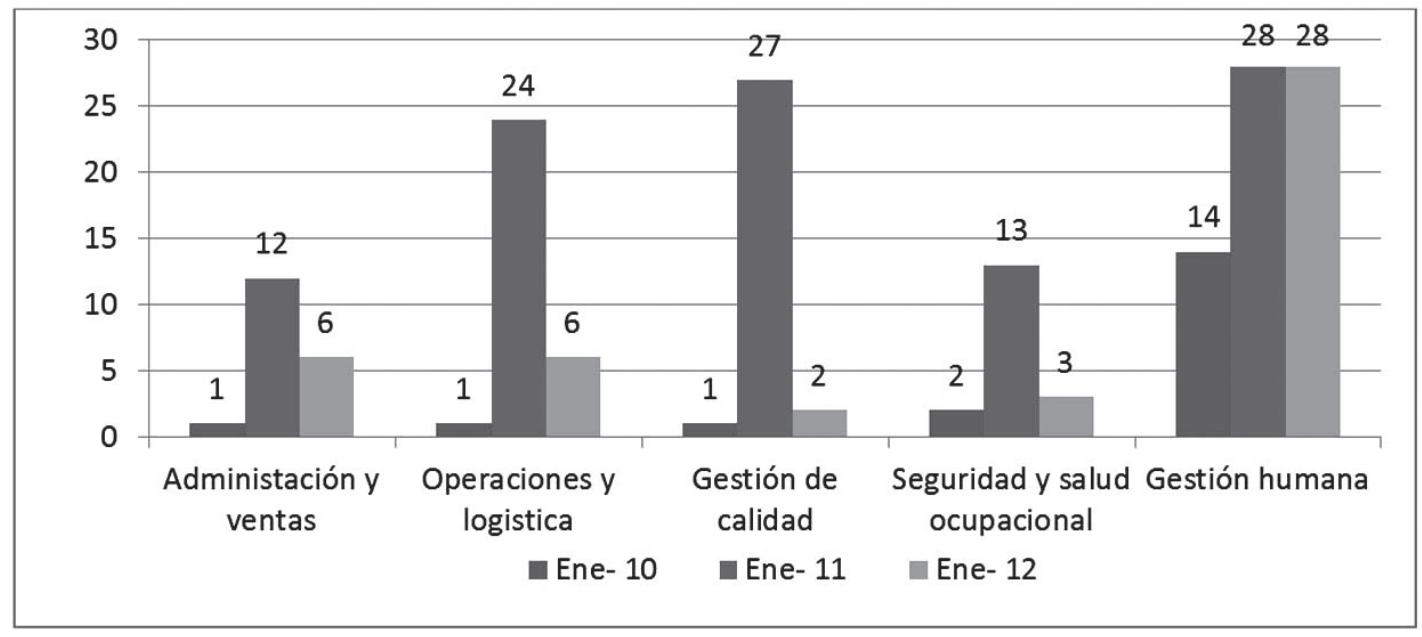

Fuente: AUSTRAL GROUP S.A.A

Sociedad Nacional de Industrias CDI - 2012 Premio Nacional a la Calidad

Los programas que están relacionados con la formación de estándares internacionales de la calidad que certifica la organización o a los requerimientos de los clientes.

Gráfico $\mathbf{N}^{\circ}$ 2. Total de Capacitaciones en horas-hombre por persona

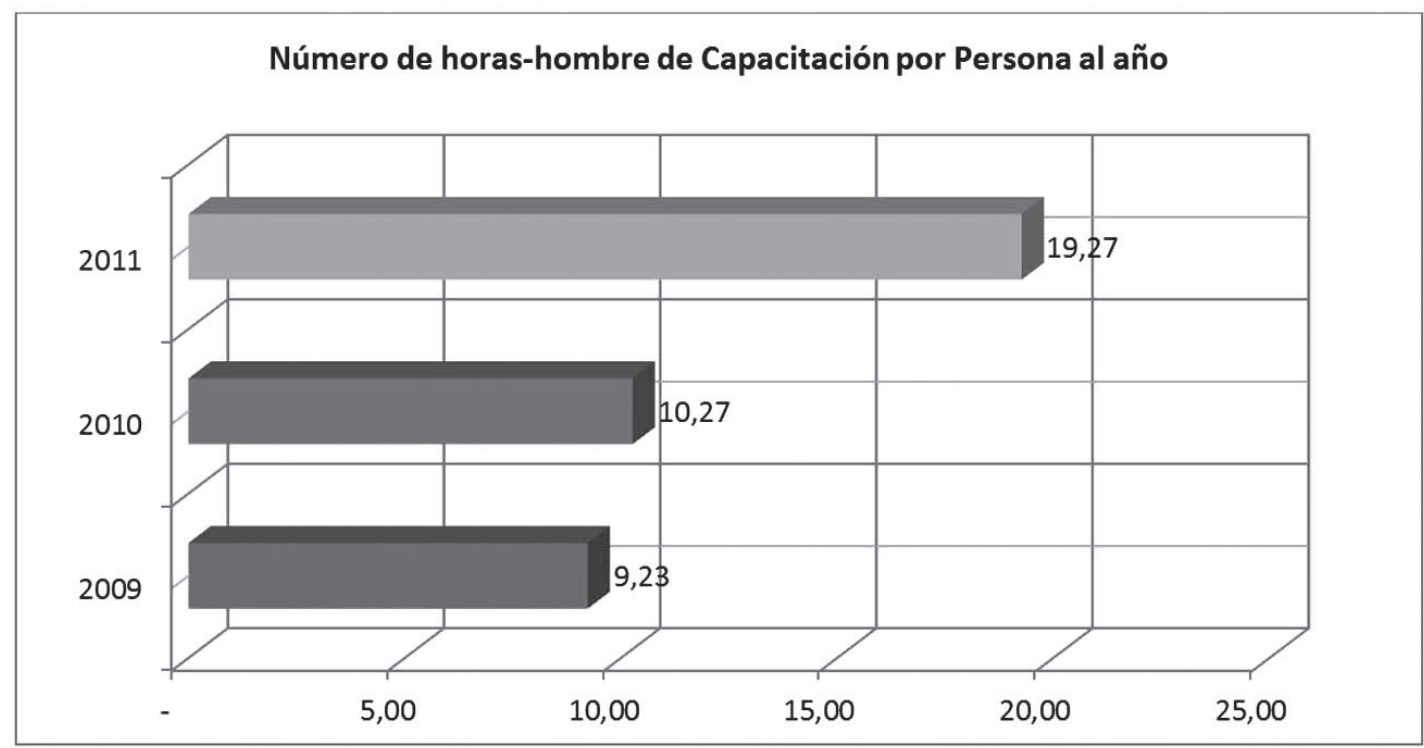

Fuente: AUSTRAL GROUP S.A.A

Sociedad Nacional de Industrias CDI - 2012 Premio Nacional a la Calidad

Estas capacitaciones fueron revisadas y coordinadas por las Jefaturas, Superintendencias y Gerencias según fue el caso, con el fin de alinear estas actividades a las metas y objetivos estratégicos. Mediante una evaluación de $360^{\circ}$ se obtuvo el nivel de eficiencia de cada participante. 
Gráfico $N^{\circ}$ 3. Número de personas/ Horas - Hombre de Capacitación - AUSTRAL

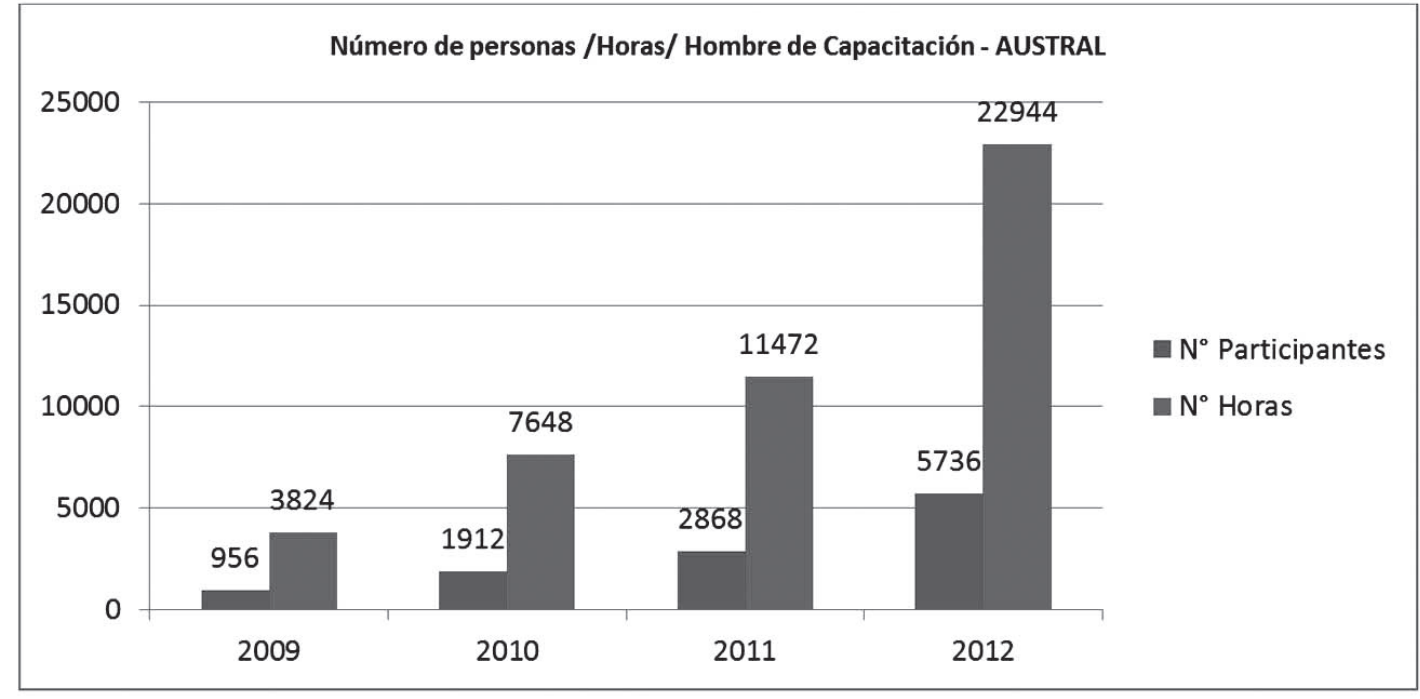

Fuente: AUSTRAL GROUP S.A.A

Sociedad Nacional de Industrias CDI - 2012 Premio Nacional a la Calidad

Cuadro $\mathrm{N}^{\circ}$ 1. Indicador de la efectividad del aprendizaje

\begin{tabular}{|c|c|c|c|c|c|c|c|}
\hline \multicolumn{8}{|c|}{ ÍNDICE DE EFECTIVIDAD DEL APRENDIZAJE } \\
\hline Área & \multirow[b]{2}{*}{ Mejora } & \multirow{2}{*}{\multicolumn{3}{|c|}{ Periodicidad de reporte anual }} & \multicolumn{2}{|c|}{ Mes de reporte: } & \\
\hline Tipo de indicador: & & & & & \multicolumn{2}{|c|}{ Mes de elaboración: } & \\
\hline $\begin{array}{l}\text { Expresión } \\
\text { Conceptual }\end{array}$ & \multicolumn{7}{|c|}{$\begin{array}{l}\text { Mide la efectividad de la capacitación mediante la nota emitida por la institución que realiza } \\
\text { la capacitación }\end{array}$} \\
\hline $\begin{array}{l}\text { Objetivo de } \\
\text { Calidad }\end{array}$ & \multicolumn{7}{|c|}{$\begin{array}{l}\text { Asegurar la efectividad del aprendizaje en la capacitación para los empleados de Austral } \\
\text { Group y Conservera de las Américas }\end{array}$} \\
\hline $\begin{array}{l}\text { Forma de } \\
\text { cálculo }\end{array}$ & \multicolumn{7}{|c|}{ X= Nota obtenida por la capacitación } \\
\hline \multirow{4}{*}{$\begin{array}{l}\text { Semáforo de } \\
\text { colores }\end{array}$} & $\begin{array}{l}\text { Línea Base } \\
\quad 2011\end{array}$ & $\begin{array}{l}\text { Meta } \\
2011\end{array}$ & $\begin{array}{l}\text { Rango de } \\
\text { Valores }\end{array}$ & \multicolumn{4}{|c|}{ Responsabilidades } \\
\hline & \multirow{3}{*}{15} & \multirow{3}{*}{17} & $17 \leq x$ & \multicolumn{2}{|c|}{ APROBAR } & \multicolumn{2}{|c|}{$\begin{array}{l}\text { GERENTE DE RECURSOS } \\
\text { HUMANOS }\end{array}$} \\
\hline & & & $15 \leq x<17$ & \multicolumn{2}{|c|}{ LOGRAR } & \multicolumn{2}{|c|}{$\begin{array}{l}\text { ANALISTA DE RECURSOS } \\
\text { HUMANOS }\end{array}$} \\
\hline & & & $x \leq 15$ & \multicolumn{2}{|c|}{ REPORTAR } & \multicolumn{2}{|c|}{$\begin{array}{l}\text { ANALISTA DE RECURSOS } \\
\text { HUMANOS }\end{array}$} \\
\hline $\begin{array}{l}\text { Factores } \\
\text { críticos }\end{array}$ & \multicolumn{7}{|c|}{$\begin{array}{l}\text { Incrementar el interés por parte del trabajador hacia la capacitación. } \\
\text { Alto nivel de responsabilidad y enfoque de la capacitación. } \\
\text { Disponibilidad de tiempo de los participantes. } \\
\text { Asignar a los expositores con las competencias requeridas en un alto nivel. } \\
\text { Asistir puntualmente a la capacitación. } \\
\text { Contar con las instalaciones y materiales adecuados para la capacitación. } \\
\text { Determinar el horario de la capacitación que facilite la asistencia. }\end{array}$} \\
\hline $\begin{array}{l}\text { Fuentes de } \\
\text { información }\end{array}$ & \multicolumn{7}{|c|}{$\begin{array}{l}\text { Notas obtenida a través de instituciones, universidades, proveedores que generen } \\
\text { capacitaciones que conllevan a un aprendizaje y apliquen una evaluación. }\end{array}$} \\
\hline
\end{tabular}

Fuente: AUSTRAL GROUP S.A.A

Sociedad Nacional de Industrias CDI - 2012 Premio Nacional a la Calidad 
Cuadro $\mathrm{N}^{\circ}$ 2. Matriz de relación entre el potencial y el desempeño

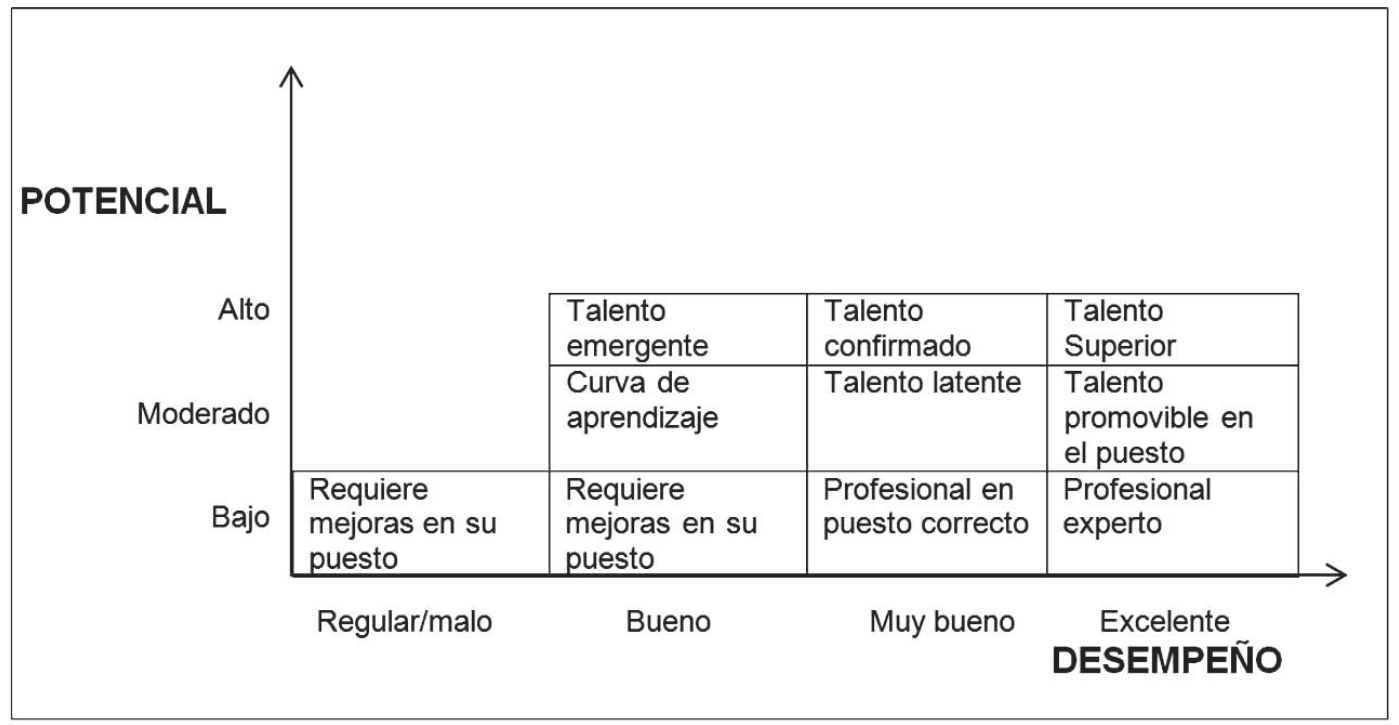

Fuente: AUSTRAL GROUP S.A.A

Categoría Producción:

TASA - Tecnológica de Alimentos S.A.

Informe de Postulación (2012), demuestra que aplica un Modelo de Gestión de desempeño en el cual utiliza capacitación por competencias "Empresa de Producción con 3479 trabajadores de los cuales $48 \%$ es personal obrero, $32 \%$ personal tripulante y el $18 \%$ es personal administrativo.

Gráfico $N^{\circ} 4$. Modelo de Gestión del Desempeño

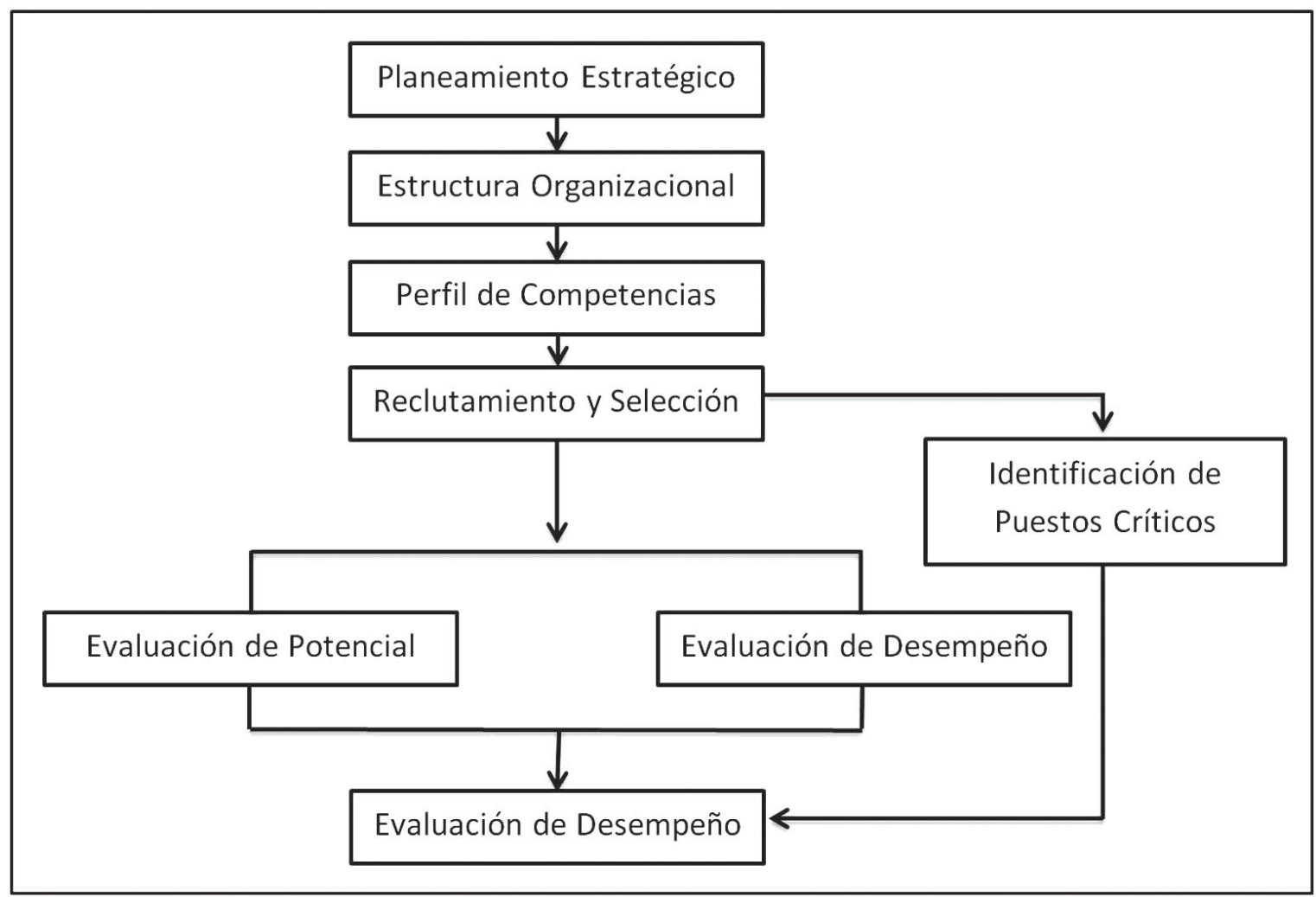

Fuente: TASA

Sociedad Nacional de Industrias CDI - 2012 Premio Nacional a la Calidad 
Informe de Postulación (2012). Esta empresa demuestra que "se gestiona la estructura y/o necesidades de personal con el Gerente General, se han realizado cambios en la estructura como la creación de la Gerencia de Investigación y Desarrollo, Gerencia de Omega 3 (nueva unidad de negocio), reestructuración de las Unidades de Pesca y Operaciones con apoyo de consultores externos), el cambio en la línea de reporte de la Gerencia de RR.HH y está a la Gerencia General. Los perfiles de puestos contienen las funciones y responsabilidades del cargo, así como, las competencias y experiencia requeridas se revisan periódicamente tienen un Modelo de Competencias que permite asegurar la adaptación a la cultura de la empresa, mejorándose el 2011 en función a los desafíos y planes estratégicos. Se identificaron 84 puestos críticos a través del establecimiento de criterios alineados al negocio y visión de la empresa, los cuales fueron aprobados por la Alta Dirección.

TASA realiza la búsqueda, contratación y retención de nuevo personal mediante el siguiente procedimiento:

Gráfico $\mathrm{N}^{\circ} 5$. Proceso de Incorporación de Nuevo Personal

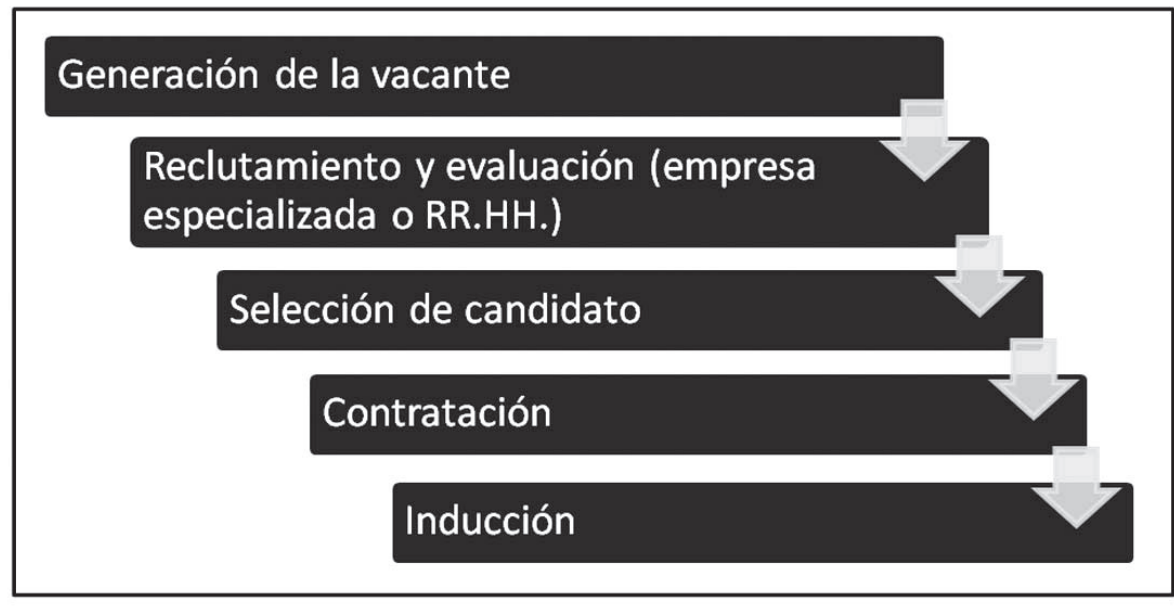

Fuente: TASA

Sociedad Nacional de Industrias CDI - 2012 Premio Nacional a la Calidad 


\section{Cuadro $\mathrm{N}^{\circ}$ 3. Objetivos alcanzados por el Personal de TASA}

\begin{tabular}{|c|c|}
\hline $\begin{array}{l}\text { Cumplir el } \\
\text { trabajo de la } \\
\text { organización }\end{array}$ & $\begin{array}{l}\text { - Los perfiles empleados para la selección y evaluación de desempeño del personal } \\
\text { establecen las funciones y responsabilidades de cada puesto. } \\
\text { - En la fase de difusión del plan estratégico se establecen y explican al personal los } \\
\text { vínculos entre los objetivos de la organización y los de sus respectivas áreas. }\end{array}$ \\
\hline $\begin{array}{l}\text { Capitalizar las } \\
\text { competencias } \\
\text { esenciales de la } \\
\text { organización }\end{array}$ & $\begin{array}{l}\text { - Los objetivosy proyectos estratégicos parten de un proceso de análisis que busca } \\
\text { capitalizar las fortalezaspara aprovechar las oportunidadesy minimizar el impacto } \\
\text { de las amenazas externas. } \\
\text { - Cuando es necesario se revisan y modifican las estructuras, perfiles, políticas u otros } \\
\text { y los cambios se difunden al personal. }\end{array}$ \\
\hline $\begin{array}{l}\text { Reforzar el } \\
\text { enfoque en los } \\
\text { clientes y en el } \\
\text { negocio }\end{array}$ & $\begin{array}{l}\text { - Se mide y gestiona la satisfacción del cliente interno y externo, siendo estos } \\
\text { indicadores clave en la evaluación de desempeño organizacional. } \\
\text { - Para la medición y análisis del desempeño organizacional se emplea un mapa } \\
\text { estratégico y un BSC que establece las relaciones de causa y efecto entre las } \\
\text { principales variables del negocio. }\end{array}$ \\
\hline $\begin{array}{l}\text { Superar las } \\
\text { expectativas de } \\
\text { desempeño }\end{array}$ & $\begin{array}{l}\text { - La aplicación de un modelo de gestión para implementar la mejora continua y la } \\
\text { innovación constante en todos los ámbitos de la organización. } \\
\text { - El proceso sistemático de la evaluación de desempeño incluye elaboración de planes } \\
\text { de desarrollo para mejorar el desempeño individual y organizacional. }\end{array}$ \\
\hline $\begin{array}{l}\text { Abordar sus } \\
\text { desafíos } \\
\text { estratégicos y } \\
\text { planes de } \\
\text { acción }\end{array}$ & $\begin{array}{l}\text { - Los procesos de despliegue de objetivosy control de gestión descritos en el punto } \\
2.2 \text { aseguran la implementación de los planes para cumplir los objetivos estratégicos } \\
\text { y alcanzar la visión. } \\
\text { - Atraer, retener y comprometer con los objetivosy la cultura al personal más } \\
\text { calificado de la industria es el elemento clave para lograr dichos objetivos. }\end{array}$ \\
\hline
\end{tabular}

Fuente:TASA

Categoría: Sector Público:

PETRÓLEOS DEL PERÚ S.A. - PETROPERÚ S.A. - OPERACIONES TALARA (OTL)

Esta empresa Líder - Grande del Sector Público (CDI 2012), demuestra que "teniendo 589 traba- jadores realiza Gestión por desempeño basado en las competencias esenciales alineadas a su misión y visión establecida al 31 de diciembre del 2011 en su PLES 2012 - 2016. El64\% de trabajadores pertenecen a las áreas operativas, y el 365 a las áreas administrativas realiza en su personal. 


\section{Gráfico $N^{\circ}$ 6. Propósito Misión, Valores y Competencias Esenciales}

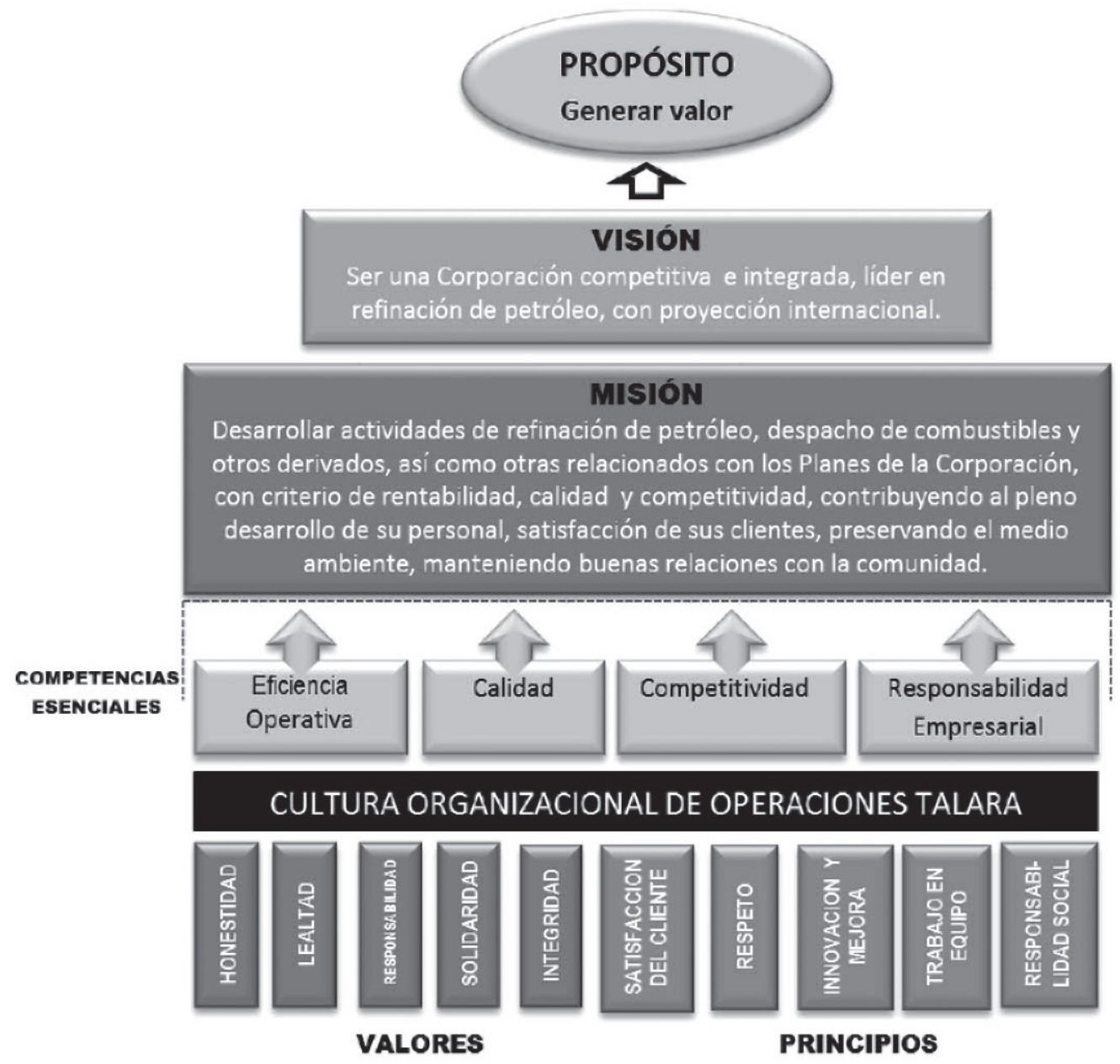

Fuente: PETRÓleOS DEL PERÚ S.A.- PETROPERÚ S.A. - OPERACIONES TALARA (OTL). Sociedad Nacional de Industrias CDI -2012 Premio Nacional a la Calidad.

La Alta Dirección $(\mathrm{AD})$ crea una organización sostenible cumpliendo y mejorando el proceso de implementación del PLES (Plan Estratégico), el cual se revisa anualmente y se concreta en objetivos y metas de seguimiento periódico, lo cual se detalla en la siguiente tabla: 


\section{Cuadro $\mathrm{N}^{\circ} 4$. Tabla de Mecanismos de logro}

\begin{tabular}{|c|c|}
\hline ASPECTO & MECANISMO DE LOGRO \\
\hline $\begin{array}{l}\text { Creando un ámbito para la } \\
\text { mejora de desempeño } \\
\text { organizacional, el cumplimiento } \\
\text { de la misión y los objetivos } \\
\text { estratégicos, la innovación y el } \\
\text { desempeño del liderazgo y la } \\
\text { flexibilidad organizacional }\end{array}$ & $\begin{array}{l}\text { - Para la mejora del desempeño organizacional se establecen metas por dependencias } \\
\text { alineados a los OE de las organización (Ver subcriterio 2.1.a. (2)). Asimismo, ha } \\
\text { implementado un Sistema Integrado de gestión (ISO 9001, ISO } 14001 \text { y OSHAS 18001), } \\
\text { el cual tiene una Estructura Organizacional, según la figura 1.1.1. } \\
\text { - Para el cumplimiento de la misión y objetivos estratégicos, se analizan los avances del } \\
\text { PLES en forma mensual. La AD toma acciones respecto de los objetivos que tienen } \\
\text { menos avance. } \\
\text { - Para la innovación, se establecen canales de comunicación abierta, a través del PLAN } \\
\text { DE SUGERENCIAS y del PLAN DE MOTIVACIÓN DEL PERSONAL EN AHORRO DE } \\
\text { ENERGÍA. } \\
\text { - Con relación al desempeño dI liderazgo, la AD promueve el “empowerment” en todos } \\
\text { los Puestos de los Empleados Técnicos Administrativos (ETA). } \\
\text { - Respecto de la flexibilidad organizacional, la AD revisa los resultados del PLES, verifica } \\
\text { si los procesos y la estructura orgánica están alineados al cumplimiento de la misión y } \\
\text { visión,y de ser necesario gestionan los cambios respectivos. (Subcriterio 2.1.b.(1)). }\end{array}$ \\
\hline $\begin{array}{l}\text { Creando una cultura en el } \\
\text { personal que brinda a los } \\
\text { clientes una experiencia } \\
\text { consistentemente positiva y } \\
\text { favorece el compromiso de ellos }\end{array}$ & $\begin{array}{l}\text { - La AD promueve cursos de capacitación: Atención al cliente, Aseguramiento de la } \\
\text { Calidad, Control metrológico, otros. } \\
\text { - OTL tiene implementado y certificado un Sistema de gestión de la Calidad (ISO } \\
\text { 9001:2008) en su instalación Portuaria y acreditado un sistema de gestión de Calidad } \\
\text { en Laboratorio (ISO/IEC 17025:2005). } \\
\text { - OTL tiene un Programa de Pasantías del Personal en las otras Unidades de Negocio de } \\
\text { la Corporación. }\end{array}$ \\
\hline $\begin{array}{l}\text { Creando un ámbito para el } \\
\text { aprendizaje organizacional y del } \\
\text { personal }\end{array}$ & $\begin{array}{l}\text { A través de la gestión de aprobación de: } \\
\text { - Programa Anual de Capacitación (PAC) } 2009-2011 \text { y 2012-2014, que considera dictado } \\
\text { de cursos in-house y en el extranjero. } \\
\text { - Pasantías en otras operaciones. } \\
\text { - Plan de Ayuda Educacional (PAE). (Subcriterio 5.1.b. (2)"beneficios"). }\end{array}$ \\
\hline
\end{tabular}

Fuente: PETRÓLEOSDEL PERÚ S.A.- PETROPERÚ S.A. - OPERACIONES TALARA (OTL). Sociedad Nacional de Industrias CDI - 2012 Premio Nacional a la Calidad.

Los planes clave de personal como parte de los Planes de Acción para el logro de los OE de corto y largo plazo, están enfocados en la necesidad de capacitar al personal, contratar personal adicional y procurar la satisfacción laboral tomando en cuenta los cambios potenciales de las necesidades de aptitud, mediante la capacitación especializada y el entrenamiento en el País o en el extranjero Plan 2012 - 2014.

\section{AÑO 2010: Premio Nacional a la Calidad del Perú y Medalla líder en Calidad Cate- GORÍA Oro:}

Categoría: Comercio y Servicios:

TELEATENTO DEL PERÚ S.A.C.

Informe de Postulación (2010) demuestra que el logro del objetivo de diferenciación vía calidad y generación de valor en sus estrategias planteadas, 4 fueron los aspectos principales, de los cuales uno de ellos fue la de Iniciativas globales de personas que fue- ron desarrolladas de manera conjunta y coordinada que consistió en a) Academia de Atento: Consistente de un Programa de Formación especializada para Supervisores y Coordinadores con mando medio para desarrollar liderazgo actual y prepararlos para el crecimiento profesional, alineado conocimiento y competencias individuales a la estrategia del negocio, b) Puntos Max: Modelo de premiación personal en el que se le da puntos para canje de artículos motivando el mejor desempeño, c)Atento Rally era dirigido a todos sus colaboradores sin importar el rol dentro de la organización, buscaba reforzar relación entre los colaboradores, la comunidad, la marca y los valores de la empresa. d) Buena Onda: voluntariado y apoyo para plataformas de atención de llamadas telefónicas.

Hemos podido apreciar que las empresas antes mencionadas en los años 2012 y 2010 respectivamente; pero existen más, y continúan en búsqueda de la excelencia, evidenciándose progreso continuo. 
Gráfico $N^{\circ} 7$. Modelo de Mapa Estratégico de Teleatento del Perú SAC - Empresa de Comercio y Servicios

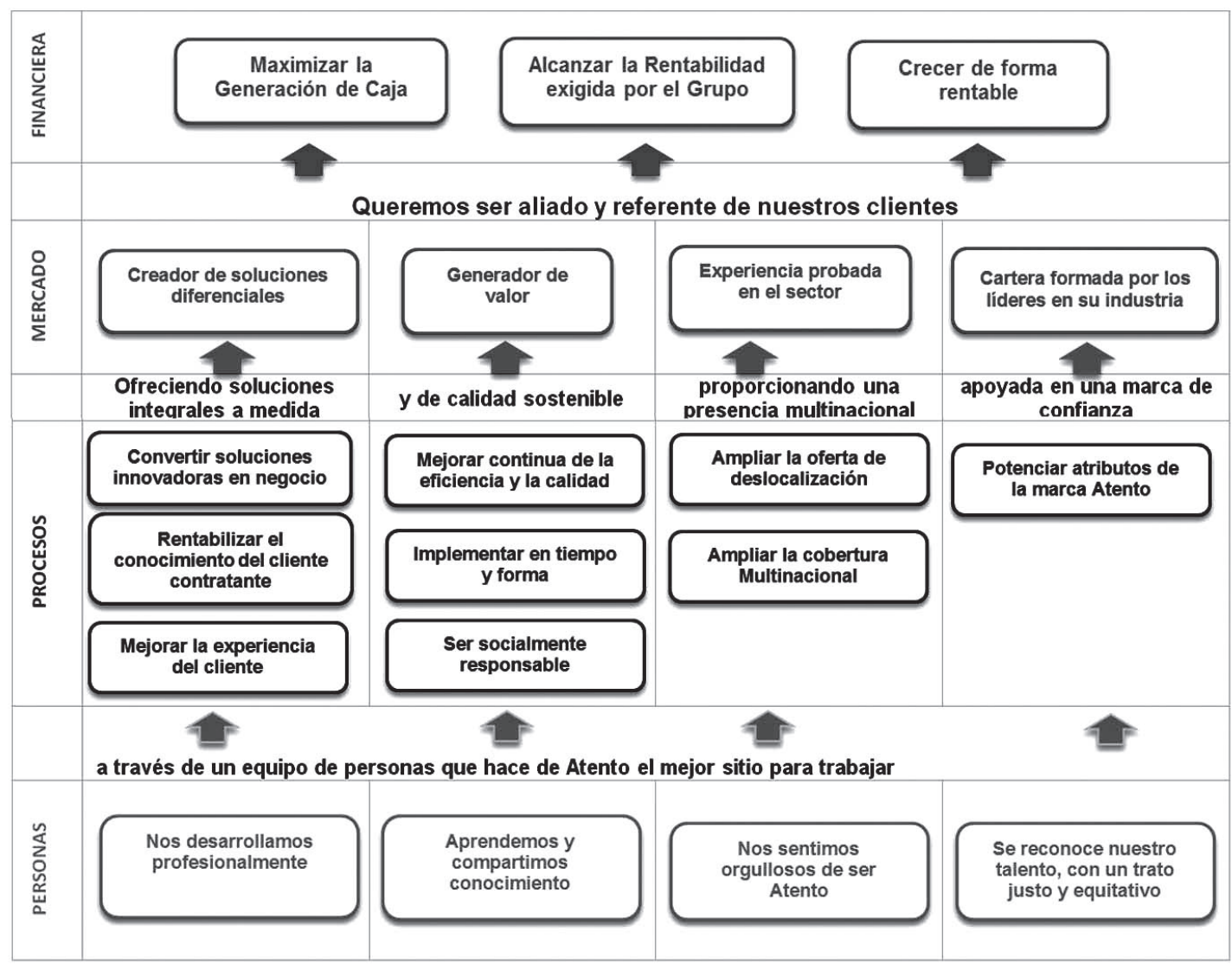

Fuente: Informe de Postulación (2010)

Sociedad Nacional de Industrias CDI - 2010 Premio Nacional a la Calidad

\section{CONCLUSIONES}

1. El Balance Scorecard y la metodología de Capacitación por Competencias son de gran importancia para obtener resultados óptimos en la inversión de capacitación de las personas, la recuperación de la rentabilidad y logro del alineamiento organizacional para cumplir las metas y objetivos trazados en el Plan Estratégico.

2. La visión y misión de la Empresa deben ser puntos de partida para trazar el Balance Scorecard y luego de realizar el análisis FODA, establecer el Cuadro de la Cadena de Valor para determinar los departamentos que generan valor a la empresa.

3. Se debe hacer una medición inicial que sirva de base para ir completando la brecha que existirá con lo que queremos alcanzar, según el Plan Estratégico de la Empresa.
4. Para realizar la Capacitación por Competencias es necesario basarnos en una metodología de éxito regional e internacional, como es el caso de Martha Alles (2010) en su obra La Trilogía, la cual ha sido aplicada también en empresas peruanas con éxito.

5. La Capacitación por Competencias es planificada en un Plan Estratégico y evaluada por $360^{\circ}$.

6. La Capacitación por competencias es trazada por un alineamiento en el Balance Scorecard para cumplir el Plan estratégico de la empresa.

7. La Capacitación por Competencias es continua, debe ser evaluada durante la capacitación y realizar un seguimiento después en la aplicación a la necesidad de la empresa. 


\section{REFERENCIAS BIBLIOGRÁFICAS}

1. Alles, M. (2009). Trilogía. Diccionario de Competencias, 3(1). (1aed). Buenos Aires: Granica.

2. Alles, M. (2009). Trilogía. Diccionario de Comportamientos, 3(2). (1aed). Buenos Aires: Granica.

3. Alles, M. (2009). Trilogía. Diccionario de Preguntas, 3(3). (1aed). Buenos Aires: Granica.

4. Certo, S. (2001). Administración Moderna (8a ed.). Colombia: Prentice Hill.

5. Chiavenato, I. (2000). Administración de Recursos Humanos. (5aed). Colombia: Mc Graw Hill Interamericana S.A.

6. Chiavenato, I. (2002). Gestión del talento Humano. (1aed). Colombia: Mc Graw Hill Interamericana S.A.

7. Domínguez, L. (2003). Elaboración del perfil de competencias del especialista WEB. (Tesis inédita de maestría). Instituto Superior Politécnico José Antonio Echevarría. Cuba.

8. Ivancevich, J. (2005). Administración de recursos humanos. (1aed). México: Mc Graw Hill.
9. Kaplan, R y Norton, D. (2004). Mapas estratégicos-Convirtiendo activos intangibles en resultados tangibles. Barcelona, España: Gestión 2000.

10. Bohlander, G. y Snell, S. (2001). Administración de Recursos Humanos (12aed). México: Thomson.

\section{REFERENCIAS DE EVENTOS}

1. Centro de Desarrollo Industrial - CDI (2013) "Premio Nacional a la Calidad del Perú y Medalla Líder en calidad Categoría Oro”, Lima-Perú.

\section{REFERENCIAS ELECTRÓNICAS}

1. Pineda, P. (2000), Evaluación del impacto de la formación en las organizaciones. Recuperado de http://ddd.uab.es/pub/ educar/0211819Xn27p119.pdf

2. Pinochet, C. Enfoque por Competencias Laborales en el Trabajo, la Formación y la Capacitación. Recuperado de http://www.igtchile.cl/ rec/competencias.pdf 